\title{
MUDANÇAS CLIMÁTICAS E A FRUTICULTURA ${ }^{1}$
}

\author{
GABRIEL CONSTANTINO BLAIN ${ }^{2}$
}

RESUMO - O objetivo do estudo é descrever evidências estatísticas de mudanças climáticas que podem impactar as atividades agrícolas, em especial a fruticultura. Embora tenham sido utilizados diversos estudos realizados em distintas partes do globo, foi dada especial atenção aos conduzidos sob as condições climáticas do Estado de São Paulo, Brasil. Nas séries de temperaturas mínimas atmosféricas, são encontradas significativas tendências de aquecimento. Contudo, nenhuma elevação consistente pode ser verificada nos valores de temperatura máxima do ar. As análises estatísticas também não têm sido capazes de indicar uma coerência espacial na variabilidade temporal associada à precipitação pluvial. Entretanto, alguns resultados estatísticos, obtidos para o Estado de São Paulo, parecem indicar um recorrente atraso na retomada da estação chuvosa. Termos de indexação: temperatura do ar, precipitação pluvial, tendências.

\section{CLIMATE CHANGE AND THE FRUIT CULTURE}

\begin{abstract}
The aim of the study is to describe statistical evidences of climate changes that may impact the agricultural activities, mainly the fruit culture. The evidences were obtained from several studies carried out throughout the world. However, it was given special focus to the climate conditions of the State of São Paulo, Brazil. It has been observed significant increasing trends in the minimum air temperature over several regions of the Globe. It has also been observed no consistent trend in the maximum air temperature. Concerning rainfall events, the statistical analyses have shown a lack of spatial coherence in the temporal changes of this meteorological variable. For the State of São Paulo, it was verified some statistical results that may be linked with a recurrent delay in the resumption of the rainy season.
\end{abstract}

Index terms: air temperature, rainfall, trends.

\section{INTRODUÇÃO}

Atualmente, o tema aquecimento global e a consequente alteração nos padrões climáticos do planeta encontram-se, certamente, entre uma das principais preocupações humanas. Segundo o relatório do Painel Intergovernamental de Mudanças Climáticas (IPCC, 2007), o termo "mudança climática" refere-se a alterações nos padrões do clima, que podem ser identificadas por meio de variações persistentes por longos períodos, da ordem de décadas, na média aritmética e/ou em outras medidas de sua variabilidade. Sob o ponto de vista agrometeorológico, e em especial da fruticultura, infere-se que as atuais tendências climáticas podem modificar a intensidade e a frequência de ocorrência de eventos de tempo e clima, prejudicando, com isso, a produção agrícola. Nesse aspecto, cita-se que, segundo Zullo et al. (2006), devido à importância da agricultura para a economia brasileira, as pesquisas de climatologia agrícola devem, entre outros fatores, considerar os impactos relacionados ao aquecimento global, descritos em trabalhos como os do IPCC (2001). Assim, estabele- cendo uma analogia com essa afirmação de Zullo et al. (2006), pode-se indicar que, dada a importância da fruticultura para o setor agronômico, os atuais estudos de estatística agrometeorológica devem ter como uma de suas metas o fornecimento de informações de tendências climáticas que possam, por sua vez, ampliar a base científica de trabalhos voltados à mitigação de adversidades climáticas associadas à produção de frutas.

Neste trabalho, são apresentados alguns resultados de detecção de tendências climáticas obtidos em diversas partes do Globo terrestre, com ênfase às alterações registradas nas séries meteorológicas do Estado de São Paulo. Espera-se, em especial, fornecer informações relevantes para as pesquisas de fruticultura.

\section{ANÁLISES DO CLIMA GLOBAL E SUAS POSSÍVEIS RELAÇÕES COM PARÂME- TROS AGROMETEOROLÓGICOS}

Dentre os trabalhos realizados sob o tema mudança climática, os relatórios do IPCC ocupam

\footnotetext{
${ }^{1}$ Resumo Sinfruit 213 - Simpósio Internacional de Fruticultura - Avanços na Fruticultura (17 a 21 Outubro)

${ }^{2}$ Eng. Agro., Instituto Agronômico, C.P. 28, 13012-970, Campinas-SP. E-mail: gabriel@iac.sp.gov.br
} 
posição de destaque. De acordo com o IPCC (2007), o aumento médio da temperatura do ar atmosférico é inequívoco, sendo que onze dos últimos doze anos (1995 a 2006) estão entre os mais quentes do período de 1850 a 2006. Ainda segundo esse relatório, foi observado na temperatura média global aumento linear de aproximadamente $0,13^{\circ} \mathrm{C}$ por década nos últimos 50 anos (1957 a 2006). Essa elevação é, aproximadamente, duas vezes superior à observada no período de 1850 a 1956.

Richards (1993) também afirmou que a temperatura global aumentou desde o final do século XIX. De forma similar ao relatório do IPCC (2007), esse autor indicou que a elevação na concentração do $\mathrm{CO}_{2}$ atmosférico se apresenta como principal fator responsável por esse aumento. Karl et al. (1999), utilizando dados de diversas partes do globo, indicaram tendência global de elevação no número de dias "excessivamente quentes" (extremely hot days) observados em cada ano. Resultados semelhantes a esses foram apontados por Manton et al. (2001), com base em dados de 15 países do sudeste asiático e do sudeste do Oceano Pacífico. Alexander et al. (2006), ao analisar dados globais extremos de temperatura atmosférica na escala diária, identificaram, em 70\% das regiões avaliadas, significativa elevação nas temperaturas noturnas.

Após analisar dados meteorológicos do continente sul-americano, Vincent et al. (2005) observaram tendências de elevação ligadas principalmente à temperatura noturna. Segundo esses autores, as estações em que foram detectadas tendências significativas, concentram-se, especialmente, nas costas leste e oeste da América do Sul. Utilizando dados de temperatura do ar e de precipitação pluvial de seis regiões do Rio Grande do Sul, Sansigolo e Kayano (2010) detectaram tendências significativas em índices associados à temperatura mínima atmosférica (escalas anual e sazonal), com taxas de elevação próximas às apontadas pelos relatórios do IPCC. Em contrapartida, para índices associados às temperaturas máximas atmosféricas, Sansigolo e Kayano (2010) descreveram tendências climáticas significativas apenas para o período do verão. Em adição, ressalta-se que essa última alteração foi associada a uma diminuição (resfriamento) da ordem de $-0,6^{\circ} \mathrm{C}$ a cada100 anos.

Dufek e Ambrizzi (2006) indicaram que, no Estado de São Paulo, há indícios de tendências para uma condição atmosférica mais quente. Os autores descrevem que, particularmente para as regiões norte e central do Estado, essa elevação associou-se à diminuição de dias frios nos anos de 1990 a 2002, sendo mais severa no período de inverno. Este trabalho também recomendou que uma análise do crescimento urbano e das mudanças no uso do solo, assim como seus impactos no clima local são necessários a fim de descrever em detalhes as diferenças gerais obtidas entre os resultados de cada estação meteorológica. A possível influência das chamadas ilhas de calor urbano nas séries meteorológicas do Estado de São Paulo também é indicada por Blain et al. (2009a) e Blain (2010 e 2011a). Contudo, após analisar os estudos de Blain et al. (2007) e Blain (2009, 2010 e 2011a), torna-se possível inferir que os indícios de alteração climática associados às maiores significâncias estatísticas (menores probabilidades de ocorrência do erro tipo I) são frequentemente encontrados nas séries compostas pela temperatura mínima do ar.

Com base nesses diversos trabalhos voltados à análise do clima, em diversas partes do globo terrestre, é razoável pressupor que o aquecimento global esteja agindo de forma significativa sobre as temperaturas mínimas atmosféricas (observadas ao longo do período noturno). Dentre as diferentes implicações agrometeorológicas que essa (pressuposta) característica climática poderá acarretar, é oportuno destacar que espécies frutíferas de clima temperado apresentam um período de repouso invernal fortemente condicionado pelas condições climáticas vigentes. Para cada novo ciclo vegetativo ser iniciado, é necessário que determinada espécie criófila sofra a ação de baixas temperaturas. A "quantidade de frio" requerida para o término do denominado repouso invernal é variável para cada espécie ou variedade. Com isso, estabelecendo (novamente) uma analogia com as afirmações de Zullo et al. (2006), parece ser bastante relevante que as pesquisas da fruticultura abordem aspectos que minimizem os efeitos associados à possível redução climática do número de horas de frio acumulados ao longo de um ciclo agrícola.

Outro fenômeno climático importante para o setor agrícola é a chamada geada. Sob o aspecto agronômico, esse evento extremo é usualmente relacionado ao congelamento de tecidos vegetais causado pela baixa temperatura atmosférica. Essa adversidade agrometeorológica não é, necessariamente, associada à deposição de gelo em superfície, sendo que, em relação à sua gênese, os danos agrícolas podem ser causados por ventos (geada de adveção) ou pelo intenso resfriamento observado no sistema superfície-atmosfera-planta (geada de radiação). Ainda sob a visão agronômica, ressaltam-se os trabalhos pioneiros de Camargo (1963, 1972), voltados ao entendimento e à mitigação do referido fenômeno. Conforme indicado em Camargo (1972), no Brasil (em latitudes próximas ao intervalo $23-27^{\circ} \mathrm{sul}$ ), esse fenômeno é potencialmente danoso para culturas tro- 
picais perenes, como cítrus e bananeira. Para latitudes superiores a $27^{\circ}$ sul, as chamadas geadas precoce e tardia podem afetar fases de frutificação e floradas, respectivamente.

Sob a hipótese de que as elevações (médias) nas temperaturas atmosféricas noturnas, descritas pela literatura científica, devem ser incorporadas na estimação da probabilidade de ocorrência associada a valores de temperatura mínima extrema que podem causar danos às culturas, Blain (2011b) desenvolveu um modelo matemático, fundamentalmente baseado na distribuição geral dos valores extremos, com parâmetros estimados em função da covariável tempo. Em outras palavras, esse autor propôs, para a região de Campinas-SP, o uso de uma função paramétrica capaz de: (i) detectar a presença de tendências climáticas associadas às temperaturas atmosféricas mínimas extremas (Tminabs), e (ii) avaliar a influência da referida alteração climática sobre a estrutura de probabilidade da variável sob análise.

Segundo Blain (2011b), embora o modelo tenha indicado um aumento médio nos valores de Tminabs (1890-2010), não foi possível concluir que a região de Campinas está atualmente livre da ocorrência de geadas. O modelo também revelou uma tendência de elevação na variância dos dados. Sob o aspecto agronômico, conclui-se que medidas de planejamento e mitigação dos efeitos das geadas não devem ser abandonadas, uma vez que esse aumento nas medidas de dispersão da referida variável pode estar associado a valores (futuros) de temperaturas atmosféricas potencialmente prejudiciais ao setor agrícola e, em especial, à fruticultura. Na Tabela 1, é apresentada (de forma simplificada) a projeção das probabilidades de ocorrência associadas aos valores de Tminabs obtidas com base no modelo proposto por Blain (2011b).

Além da temperatura atmosférica, mudanças climáticas associadas à precipitação pluvial (PRE) podem, evidentemente, ter profundas implicações agronômicas devido à possibilidade de redução na disponibilidade hídrica às culturas. Para Blain e Pires (2011), mudanças ou variações nos padrões climáticos globais podem reduzir as trocas de fluidos (tais como, solução de sais minerais e componentes orgânicos, oxigênio e gás carbônico) no sistema solo-planta-atmosfera devido à elevação da ocorrência de estresse hídrico.

Alexander et al. (2006), ao analisarem dados globais extremos de precipitação pluvial (PRE), na escala diária, identificaram tendências de elevação nesse elemento meteorológico. Entretanto, essas últimas alterações apresentaram uma coerência espacial bastante inferior à observada nos dados de temperatura do ar (citadas anteriormente). Frei e Schar (2000), investigando tendências climáticas em eventos de PRE, na região dos Alpes europeus, detectaram, no outono e inverno, significativa elevação na frequência de ocorrência de totais desse elemento meteorológico, com valores entre $15 \mathrm{e}$ $39 \mathrm{~mm} /$ dia. Nos casos de precipitação diária mais elevada, segundo Frei e Schar (2000), não foram observadas significativas alterações.

Haylock et al. (2006), analisando tendências em valores diários de precipitação na América do Sul (1960 a 2000), afirmam que o padrão de alteração observado nos valores extremos desse elemento meteorológico é, de modo geral, o mesmo para os totais anuais. Neste trabalho, são verificadas mudanças para condições mais úmidas ("changes to wetter conditions") na parte sul do Brasil.

Sansigolo e Kayano (2010) indicaram a existência de tendências significativas de elevação apenas na PRE observada no período do verão no Rio Grande do Sul. Segundo esses autores, essa alteração apresentou uma taxa de $93 \mathrm{~mm}$ a cada 100 anos (para uma precipitação sazonal média de $367 \mathrm{~mm}$ ). Marengo et al. (2007) observaram que, no Sudeste do Brasil, o total anual de precipitação parece não ter sofrido alteração significativa nos últimos 50 anos. Corroborando essa última indicação, Blain et al. (2007), Blain et al. (2009b) e Blain (2009) apontam, para o Estado de São Paulo, a existência de tendências (com baixa significância estatística) de elevação nos totais mensais desse elemento meteorológico, especialmente concentrada no mês de maio. Para Blain (2009), a principal característica da PRE mensal do referido Estado é sua elevada variabilidade temporal, sob a qual meses extremamente úmidos podem preceder ou serem precedidos por meses extremamente secos.

Embora a relevância agrícola de trabalhos restritos à escala mensal seja apoiada por afirmações como as de Cargnelutti Filho et al. (2010), de que a precipitação pluvial é importante para o adequado planejamento das atividades agropecuárias, a análise de totais mensais de PRE permanece distante dos interesses agrometeorológicos, tendo em vista que períodos de, por exemplo, 10 ou 20 dias, em que não há observações de eventos de chuva, podem ter impactos significativos na produção agrícola, dependendo da época ou estádio fenológico de ocorrência. Dessa forma, torna-se evidente que estudos de alterações climáticas devem também avaliar possíveis mudanças na distribuição dos totais de precipitação ocorridas ao longo das distintas fases fenológicas contidas em dado ano agrícola. Com base nessa premissa, Blain (2011c) avaliou, entre outros fatores, a presença de possíveis tendências climáticas na série de precipitação pluvial decendial, da região de abrangência da estação meteorológica de Campinas (uma das mais antigas do 
País), entre os anos de 1890 e 2009.

Utilizando métodos não paramétricos e considerando as probabilidades máximas de ocorrência do erro estatístico tipo I, usualmente adotadas na literatura (10 e 5\%), não houve indicação de marcantes tendências climáticas nas séries analisadas. Contudo, observou-se concentração de casos de tendências de queda a partir do final da época seca regional, mês de agosto, até o início do mês de novembro, pertencente à estação chuvosa (especialmente marcada para os dois últimos decêndios de outubro). Essa característica pode ser considerada um indício de mudança nos padrões do clima regional no sentido de atraso da retomada da estação chuvosa (BLAIN, 2011c).

Ainda que pouco significativos sob o ponto de vista numérico-estatístico, atrasos (climáticos) na retomada da estação chuvosa são potencialmente preocupantes para a agricultura e, em especial, para a fruticultura. Nesse aspecto, cita-se que, segundo Blain e Pires (2011), alterações pouco significativas em séries temporais de precipitação pluvial (associadas, por exemplo, a probabilidades do erro estatístico tipo I $\mathrm{p} \geq 0,10$ ) podem resultar em significativas mudanças $(p \leq 0,05)$ em parâmetros relativos à demanda hídrica das culturas, como, por exemplo, a evapotranspiração real. Nesse aspecto, ressalta-se que Blain e Pires (2011) observaram, para a localidade de Campinas, uma possível implicação agronômica do referido atraso. De acordo com esses autores, tem sido observada tendência climática de queda nos valores de parâmetros agrometeorológicos (evapotranspiração real e razão entre a evapotranspiração real e potencial) associados à disponibilidade hídrica das culturas nos dois últimos decêndios de outubro. Considerando que o florescimento de espécies frutíferas cultivadas sob clima tropical (como cítrus, por exemplo) tende a ocorrer após o início/restabelecimento do período chuvoso, torna-se de suma importância que as atuais pesquisas de estatística (agro)climatológica verifiquem se essa possível alteração na variabilidade temporal da PRE de Campinas esteja ocorrendo em demais regiões agrícolas do Estado e até mesmo do País. Por fim, ressalta-se que, dado o possível deslocamento do balanço de energia em superfície no sentido do calor sensível, essa redução da disponibilidade hídrica poderá (potencialmente) resultar na elevação da probabilidade de ocorrência de temperaturas máximas extremas prejudiciais à produtividade e à qualidade das espécies frutíferas.

TABELA 1 - Projeção das probabilidades de ocorrência associadas a valores extremos (mínimos) de tem-

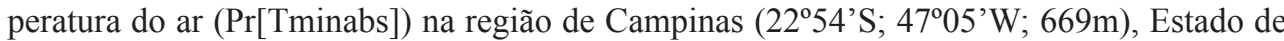
São Paulo-Brasil

\begin{tabular}{cccccc}
\hline \multirow{2}{*}{ Ano } & \multicolumn{6}{c}{$\operatorname{Pr}\left[\operatorname{Tminabs}<\mathrm{x} ; \mathrm{x}=5,4,3,2,1{ }^{\circ} \mathrm{C}\right]^{*}$} \\
\cline { 2 - 6 } & $5{ }^{\circ} \mathrm{C}$ & $4{ }^{\circ} \mathrm{C}$ & $3{ }^{\circ} \mathrm{C}$ & $2{ }^{\circ} \mathrm{C}$ & $1{ }^{\circ} \mathrm{C}$ \\
\hline $2020(\mathrm{t}=130)$ & 0.366 & 0.255 & 0.168 & 0.104 & 0.060 \\
$2030(\mathrm{t}=140)$ & 0.344 & 0.242 & 0.162 & 0.102 & 0.061 \\
$2040(\mathrm{t}=150)$ & 0.326 & 0.232 & 0.158 & 0.102 & 0.062 \\
$2050(\mathrm{t}=160)$ & 0.311 & 0.224 & 0.155 & 0.102 & 0.064 \\
\hline
\end{tabular}

*Estimativas baseadas em modelo não estacionário GEV(Tminabs $\left.; \mu_{t}, \sigma_{t}, \xi_{t}\right)$, em que $\left[\mu_{t}=3.095+0.0301 \mathrm{t}, \sigma_{t}=\exp (0.316+0.0053 \mathrm{t})\right.$, $\left.\xi_{t}=-0.177\right]$. Os "standard errors" de cada parâmetro são, respectivamente, $0.307 ; 0.005 ; 0.140 ; 0.002$ e 0.072 .

\section{SÍNTESE E CONSIDERAÇÕES FINAIS}

Sob os interesses agronômicos e, em especial, os da fruticultura, todas as preocupações associadas ao aquecimento global podem, provavelmente, ser sintetizadas em uma única sentença interrogativa: como as mudanças climáticas afetarão, ou veem afetando, a produção agrícola?

É evidente que, embora de fácil formulação, a solução dessa questão é extremamente difícil, pois envolve não apenas a elevada complexidade inerente ao sistema climático global, mas também todas as distintas respostas que cada região agrícola poderá apresentar frente a uma marcada tendência nos padrões climáticos globais. Nesse aspecto, o contínuo estudo e o consequente aprofundamento do conhe- cimento das variações temporais de tempo e clima, observadas em escala global ou regional, é apenas uma das etapas fundamentais para a mitigação das implicações agronômicas do chamado aquecimento global.

Conforme evidenciado no presente trabalho, as análises estatísticas realizadas em diversas regiões do Globo descrevem parte relevante dessa complexidade quando aplicadas, por exemplo, em longas séries meteorológicas obtidas após o registro discreto no tempo da temperatura atmosférica. Esses estudos vêm demonstrando que diferentes taxas de variação temporal, associadas a essa variável contínua, podem ser obtidas pela simples variação do horário de leitura (diurno, noturno, máxima ou mínima). Sob essa última característica, infere-se uma tendência 
climática de elevação média dos valores da temperatura atmosférica noturna, bem como uma tendência de redução na amplitude térmica diária. Verifica-se também que, mesmo regiões sujeitas a significativa elevação média dos valores da temperatura mínima do ar não podem ser consideradas livres da ocorrência de eventos agrometeorológicos extremos, como geada. Dada a referida complexidade do sistema climático, alterações em medidas de tendência central de uma série meteorológica podem ser acompanhadas por mudanças em suas medidas de dispersão. Por fim, com base em uma das séries meteorológicas mais antigas do Brasil, também foram observadas alterações na distribuição temporal da precipitação pluvial, associadas a implicações agrometeorológicas, ainda que não tenha ocorrido nenhuma mudança, com elevada significância estatística, nos totais mensais desse elemento meteorológico.

\section{AGRADECIMENTO}

$\mathrm{O}$ autor cordialmente agradece à Dra. Rose Mary Pio, do Instituto Agronômico, pelo incentivo à realização deste trabalho.

\section{REFERÊNCIAS}

ALEXANDER, L.V.; ZHANG, X.; PETERSON, T.C.; CAESAR, J.; GLEASON, B.; TANK, A.M.G; HAYLOCK, M.; COLLINS, D.; TREVIN, B.; RAHIMZADEH, F.; TAGIPOU, A.; RUPA KUMAR, K.; REVADEKAR, J.; GRIFFITHS, G.; VINCENT, L.; STEPHENSON, D.; BURN, J.; AGUILLAR, E.; TAYLOR, M.; NEW, M.; ZHAI, P.; RUSTICUCCI, M.; VASQUEZ-AGUIRRE, J.L. Global observed changes in daily climate extremes of temperature and precipitation. Journal of Geophysical Research, Washington, v.111, D05109, 2006.

BLAIN, G.C.; PIEDADE, S.M.S.; CAMARGO, M.B.P.; GIAROLLA, A. Distribuição temporal da precipitação pluvial mensal observada no Posto Meteorológico do Instituto Agronômico, em Campinas-SP. Bragantia, Campinas, v. 66, n.2, p. 347-355, 2007.

BLAIN, G.C. Considerações estatísticas relativas a oito séries de precipitação pluvial da Secretaria de Agricultura e Abastecimento do Estado de São Paulo. Revista Brasileira de Meteorologia, São José dos Campos, v.24, n.1, p.12-23, 2009.
BLAIN, G.C.; KAYANO, M.T.; CAMARGO, M.B.P.; LULU, J. Variabilidade amostral das séries mensais de precipitação pluvial em duas regiões do Brasil: Pelotas-RS e Campinas-SP, Revista Brasileira de Meteorologia, São José dos Campos, v.24, n.1, p.1-11, 2009 b.

BLAIN, G.C. Séries anuais de temperatura máxima média do ar no Estado de São Paulo: variações e tendências climáticas Revista Brasileira de Meteorologia, São José dos Campos, v.25, n.1, p.114-124, 2010.

BLAIN, G.C.; PICOLI, M.C.A.; LULU, J. Análise estatística das tendências de elevação nas séries anuais de temperatura mínima do ar no Estado de São Paulo. Bragantia, Campinas, v.68, n.3, p.807815, 2009a.

BLAIN, G.C; PIRES, R.C.M. Variabilidade temporal da evapotranspiração real e da razão entre evapotranspiração real e potencial em Campinas, Estado de São Paulo, Bragantia, Campinas, v. 70, n.3, (prelo) $2011 \mathrm{~b}$.

BLAIN, G.C. Considerações estatísticas relativas a seis séries mensais de temperatura do ar da Secretaria de Agricultura e Abastecimento do Estado de São Paulo.Revista Brasileira de Meteorologia, São José dos Campos, v.26, n.2, p. 279-296, 2011 ${ }^{\mathrm{a}}$.

BLAIN, G.C. Totais decendiais de precipitação pluvial em Campinas-SP: persistência temporal, periodicidades e tendências climáticas. Ciência Rural, Santa Maria, v.41, n.5, p. 789-795, 2011c.

BLAIN, G.C. Incorporating climate trends in the stochastic modeling of extreme minimum air temperature series of Campinas, State of São Paulo, Brazil, Bragantia, Campinas, v. 70, (prelo) 2011b.

CAMARGO, A.P. Instruções para o combate à geada em cafezais. Campinas, IAC, 1963. 18p. (Boletim Técnico 130)

CAMARGO, A.P. Apontamentos de agrometeorologia. Apostila da FAZMCG, Espírito Santo do Pinhal, 1972.

DUFEK, A.S.; AMBRIZZI, T. Variabilidade climática da temperatura no Estado de São Paulo. In: CONGRESSO BRASILEIRO DE METEOROLOGIA, 14., 2006, Florianópolis. Anais...Florianópolis: SBMET, 2006. 1 CD-ROM. 
FREI, C.; SCHAR, C. Detection probability of trends in rare events: Theory and application to heavy precipitation in the Alpine region. Journal of Climate, Washington, v. 14, p. 1568-1584, 2000.

HAYLOCK, M.R.; PETERSON, T.C.; ALVES, L.M.; AMBRIZZI, T.; ANUNCIAÇÃO, Y.M.T.; BAEZ, J.; BARROS, V.R.; BERLATO, M.A.; BIDEGAIN, M.; CORONEL, G.; CORRADI, V.; GARCIA, V.J.; GRIMM, A.M.; KAROLY, D.; MARENGO, J.A.; MARINO, M.B.; MONCUNILL, D.F.; NECHET, D.; QUINTANA, J.; REBELLO, E.; RUSTICUCCI, M.; SANTOS, J.L.; TREBEJO, I.; VINCENT, L.A. Trends in total and extreme South American rainfall in 1960-2000 and links with sea surface temperature. Journal of Climate, Washington, v. 19, p. 1490-1512, 2006.

IPCC. Climate Change 2001: Impacts, Adaptation and Vulnerability, Contribution of Working Group 2 to the Third Assessment Report of the Intergovernmental Panel on Climate Change, HOUGHTON, JT, (Ed,), Cambridge University Press,2001.

IPCC. Climate Change 2007: The Physical Science Basis, Contribution of Working Group I to the Fourth Assessment Report of the Intergovernmental Panel on Climate Change, HOUGHTON, JT, (Ed,), Cambridge University Press, 2007.

KARL, T.R.; NICHOLLS, N.; GHAZI, A. 1999. CLIVAR:GCOS:WMO workshop on indices and indicators for climate extremes. Climatic Change Washington, v. 42, p.3-7, 1999.

MANTON, M.J.; DELLA-MARTA, P.M.; HAYLOCK, M.R.; HENNESSY, K.J.; NICHOLLS, N.; CHAMBERS, L.E.; COLLINS, D.A.; DAW, G.; FINET, A.; GUNAWAN, D.; INAPE, K.; ISOBE, H.; KESTIN, T. S.; LEFALE, P.; LEYU, C. H.; LWIN, T.; MAITREPIERRE, L.; OUPRASITWONG, N.; PAGE, C.M.; PAHALAD, J.; PLUMMER, N.; SALINGER, M.J.; SUPPIAH, R.; TRAN, V.L.; TREWIN, B.; TIBIG, I.; YEE, D. Trends in extreme daily rainfall and temperature in Southeast Asia and the South Pacific: 1961-1998. International. Journal of Climatology, Bracknell, v.21, p.269-284, 2001.
MARENGO, J.; NOBRE, C.; RAIGOZA, D.; VALVERDE, M.; PISNITCHENKO, I.A.; OLIVEIRA, J.C.M. Boletim do Projeto: uso de cenários de mudanças climáticas regionais em estudos de vulnerabilidade e adaptação no Brasil e na América do Sul (GOF-UK-CPTEC), 2007. Disponível em: $<$ www.cptec.inpe.br/mudancas_climaticas $>$.Acesso em: $1^{\circ}$ nov. 2008.

RICHARDS, G. R. Change in global Temperature: A statistical analysis, Journal of Climate, Washington, v.6, p. 556-559, 1993.

SANSIGOLO, C.A.; KAYANO, M.T. Trends of seasonal maximum and minimum temperatures and precipitation in Southern Brazil for the 1913-2006 period, Theoretical and Applied Climatology, Amsterdam, v. 101, p.209-216, 2010.

VINCENT, L.A.; PETERSON, T.C.; BARROS, V.R.; MARINO, M.B.; RUSTICUCCI, M.; CARRASCO, G.; RAMIREZ, E.; ALVES, L.M.; AMBRIZZI, T.; BERLATO, M.A.; GRIMM, A.M.; MARENGO, J.A.; MOLION. L.; MONCUNILL, D.F.; REBELLO, E.; ANUNCIAÇÃO, Y.M.T.; QUINTANA, J.; SANTOS, J.L.; BAEZ, J.; CORONEL, G.; GARCIA, J.; TREBEJO, I.; BIDEGAIN, M.; HAYLOCK, M.R.; KAROLY, D. Observed trends in indices of daily temperature extremes in South America 1960-2000. Journal of Climate, Washington, v.18, p.5011-5023, 2005.

ZULLO JR., J.; PINTO, H.S.; ASSAD, E.D. Impact assessment study of climate change on agricultural zoning, Meteorological Applications, v.13, p. 6980 Cambridge University Press, 2006 doi:10.1017/ S135048270600257X 